\title{
Inverted duplications: how many of them are mosaic?
}

\author{
Tiziano Pramparo ${ }^{1}$, Sabrina Giglio ${ }^{2}$, Giuliana Gregato ${ }^{1}$, Manuela de Gregori ${ }^{1}$, Maria Grazia \\ Patricelli $^{1}$, Roberto Ciccone ${ }^{1}$, Susi Scappaticci ${ }^{1}$, Gaetano Mannino ${ }^{3}$, Claudio Lombardi ${ }^{4}$, \\ Barbara Pirola ${ }^{2}$, Roberto Giorda ${ }^{5}$, Mariano Rocchi ${ }^{6}$ and Orsetta Zuffardi ${ }^{\star, 7}$
}

\begin{abstract}
${ }^{1}$ Biologia Generale e Genetica Medica, University of Pavia, Pavia, Italy; ${ }^{2}$ Diagnostica e Ricerca, San Raffaele Hospital, Milano, Italy; ${ }^{3}$ Azienda Ospedaliera Vimercate, Italy; ${ }^{4}$ Studio Diagnostico ECO, Vimercate, Italy; ${ }^{5}$ IRCCS La Nostra Famiglia, Bosisio Parini, Lecco, Italy; ${ }^{6}$ DAPEG Sezione di Genetica, Bari, Italy; ${ }^{7}$ Biologia Generale e Genetica Medica, University of Pavia, Pavia and IRCCS Policlinico San Matteo, Pavia, Italy
\end{abstract}

The best-known situation indissolubly linked to mosaicism is the uniparental disomy where a trisomic or monosomic zygote develops at least one cell line with $\mathbf{4 6}$ chromosomes. The mosaicism normal/abnormal cell lines may remain confined to placenta or persist in the embryo. Here, we describe a second situation that might also be indissolubly linked to a mosaic condition or at least to a confined placental mosaicism. We analysed the case of a mosaicism del(8p)/inv $\operatorname{dup}(8 p)$ found in prenatal diagnosis. We had already demonstrated that the first product of the abnormal meiotic recombination at the basis of the inv dup rearrangements is a dicentric chromosome. Its breakage leads to the formation of a deleted and an inv dup chromosome. Although we had previously assumed that the dicentric underwent a breakage at meiosis II so that the zygote inherited the inv dup chromosome, our findings and those of others indeed indicate that the dicentric may be inherited in the zygote and that it might persist as such in early postzygotic stages, then undergoing different breakages in different cells leading to different abnormal chromosomes, either deleted or duplicated. Selection versus the most viable cell line(s) results either in a confined placental mosaicism with the inv dup cell line as the only one present in the embryo or in children with both the deleted and the inv dup cell lines. Phenotype/karyotype relationships in inv dup rearrangements must also take into account the influence of the other abnormal cell line during embryogenesis.

European Journal of Human Genetics (2004) 12, 713-717. doi:10.1038/sj.ejhg.5201240

Published onlne 21 July 2004

Keywords: dicentric chromosome; inverted duplication; mosaicism

\section{Introduction}

The molecular characterization of several rearrangements interpreted as simple duplications led to the discovery that most of them were in fact inverted duplications associated with the deletion of the portion distal to the duplication. The best-known case is that of the inv dup $(8 \mathrm{p}){ }^{1,2}$ Other examples are those concerning $1 q^{3,4} 2 q,{ }^{5} 3 p^{6,7} 4 p,{ }^{8} 5 p,{ }^{9,10}$ $9 \mathrm{p},{ }^{11} 10 \mathrm{p}$ and $10 \mathrm{q},{ }^{12} 18 \mathrm{p}^{13} 18 \mathrm{q},{ }^{14} 21 \mathrm{q}^{15}$ and the $\mathrm{X}$; $\mathrm{X}$ or the

*Correspondence: Dr O Zuffardi, Biologia Generale e Genetica Medica, Via Forlanini 14, 27100 Pavia, Italy. Tel.: + 39038250 7733; Fax: + 39038 252 5030; E-mail: zuffardi@unipv.it

Received 11 March 2004; revised 22 April 2004; accepted 5 May 2004
Y;Y rearrangements leading to duplications of parts of the short or the long arm with concomitant deletion. ${ }^{16}$ It has been assumed ${ }^{1,5}$ that the first product of the abnormal meiotic recombination on the basis of this type of rearrangement was a dicentric chromosome, either $\mathrm{p} \rightarrow \mathrm{q}:: \mathrm{q} \rightarrow \mathrm{p}$ or $\mathrm{q} \rightarrow \mathrm{p}:: \mathrm{p} \rightarrow \mathrm{q}$. The dicentric, due to its intrinsic instability, would undergo an asymmetric breakage between the two centromeres leading to the final inv dup. In the case of the inv $\operatorname{dup}(8 p)$, the breakage of the original dicentric chromosome 8qter $\rightarrow$ 8p23.1::8p23.1 $\rightarrow$ 8qter occurs in different positions between the two centromeres with a preferential breakpoint at the level of the second centromere that became inactive (six out of 16 
cases). ${ }^{1}$ In no other case of autosomal inv dups two alphoid signals have been reported; this seems to be due to the fact that very large duplications are unviable or that the second centromere has not been detected due to the use of staining techniques inappropriate in highlighting the centromere. Previously, we had assumed ${ }^{1,5}$ that the dicentric's breakage occurred at the second meiotic division leading to a gamete having the inv $\operatorname{dup}(8 \mathrm{p})$ and a gamete deleted for a portion of $8 \mathrm{p}$. However, two recent papers $^{17,18}$ and an older one ${ }^{19}$ made us re-evaluate the entire situation suggesting that the breakage of the dicentric might not occur at meiosis II but that, at least in some cases, the dicentric could be inherited as such in the zygote, after which it undergoes to breakage, thus, leading to a mosaic situation of the conception's product. The mosaicism consists in a cell line containing the inv $\operatorname{dup}(8 \mathrm{p})$ and a second cell line containing either the deleted $8 \mathrm{p}$ or other products derived from the deletion of the dicentric. The finding of a new mosaic inv dup(8p) case indeed made us hypothesize that mosaicism or at least confined placental mosaicism might be the rule for all inv dup rearrangements.

\section{Case report}

A 32-year-old healthy woman was referred between the 11 th and 12th week of pregnancy for cytogenetic evaluation after abnormal fetal ultrasound findings showing subcutaneous oedema in the neck region with generalized fetal hydrops associated with heart malformation. Transabdominal and transvaginal echocardiography with colour doppler flow imaging showed atrial and ventricular septal defect, dilated left ventricle and pericardial effusion. The couple decided for the termination of pregnancy at the end of the 12th week, after the finding of the $8 \mathrm{p}$ deletion on the direct chorionic villus sample.

\section{Materials and methods}

Cytogenetic analysis was performed on a 12 -week chorionic villus sample (CVS) both after $24 \mathrm{~h}$ incubation (direct CVS) and after 11 days of culturing (cultured CVS). Other metaphases were analysed on slides obtained from other long-term cultures. FISH with subtelomeric probes was carried out following the manufacturer's (Vysis) instructions. Single- and dual-colour FISH analysis was performed as described. ${ }^{2}$ BAC DNA extraction was carried out using the PhasePrep BAC DNA kit (SIGMA). BAC clones were labelled with biotin-16dUTP and digoxigenin-dUTP by nick translation (BRL Life Technologies). Signals from biotin-labelled probes were developed using alternate layers of avidin-fluorescein isothiocyanate (avidin-FITC) and biotinylated anti-avidin. Signals from digoxin-labelled probes were developed using alternate layers of mouseantidigoxigenin, avidin-alpha-mouse and antiavidin-alpha-rabbit antibodies. Slides were counterstained with DAPI (Sigma) and mounted in Vectashield antifading medium (Vector Laboratories, USA). Signals were visualized under a Nikon E1000 microscope equipped with a cooled charge-coupled device (CCD) camera and Genikon image analysis software. Genotyping of $8 \mathrm{p}$ polymorphic loci from the DNAs of the CVS and the parents was performed by amplification with primers (Table 1) labelled with fluorescent probes (ABI 5-Fam, Hex) followed by analysis on a ABI 3100 Genetic Analyzer (Applied Biosystems). Amplifications were performed with Taq Gold (Applied Biosystems) using standard protocols. Cytogenetic analysis from peripheral lymphocytes of the parents was carried out.

\section{Results}

A deletion of the short arm of one chromosome 8 was detected in all the 26 cells that could be analysed from the direct CVS: 46,XX,del(8)(p11. 2) (Figure 1a). Analysis of 20 metaphases obtained from cultured CVS demonstrated the presence of a derivative chromosome 8 with added material on the short arm: 46,XX,der(8)add(8p) (Figure $1 \mathrm{~b}$ ); in three metaphases, the elongated $8 \mathrm{p}$ ended with fluorescent satellites (Figure 1c). FISH analysis with probes RP11-13N12 (AC011586), RP11-135I5 (AC022716) (Figure 2a) and chromosome 8 alpha-satellite demonstrated that the der(8) was a monocentric inv dup(8p). A total of 98 metaphases obtained from cultured CVS were

Table 1 Polymorphic markers used to define the inv dup (8p)

\begin{tabular}{|c|c|c|c|c|c|}
\hline Locus & Band & Position & Propositus & Mother & Father \\
\hline D8S264 & $8 p 23.3$ & 2049536 & 136.72 & $132.9 / 146.64$ & $136.80 / 138.81$ \\
\hline D8S262 & $8 p 23.2$ & 3924412 & 117.19 & 117.18 & 117.15 \\
\hline D8S1742 & $8 p 23.1$ & 6468442 & 144.73 & $131.04 / 136.89$ & $138.94 / 144.70$ \\
\hline D8S1819 & $8 p 23.1$ & 7004392 & 215.05 & 213.11 & 215.02 \\
\hline D8S1731 & $8 p 22$ & 15199182 & $218.08^{\mathrm{a}} / 238.26$ & 218.08 & $222.02 / 238.31$ \\
\hline D8S258 & $8 p 21.3$ & 20429484 & $146.61 / 148.51^{\mathrm{a}}$ & $146.68 / 148.66$ & $140.88 / 148.60$ \\
\hline D8S1786 & $8 p 21.3$ & 22785956 & $210.25 / 216.06 / 224.04$ & $210.28 / 223.99$ & $210.32 / 216.08$ \\
\hline D8S1752 & $8 p 21.2$ & 23022206 & $144.03 / 145.73 / 150.00$ & $143.97 / 149.89$ & $145.93 / 147.99$ \\
\hline D8S382 & $8 p 21.2$ & 26305810 & 317.80 & 317.81 & 317.79 \\
\hline
\end{tabular}

a The allele is duplicated. 

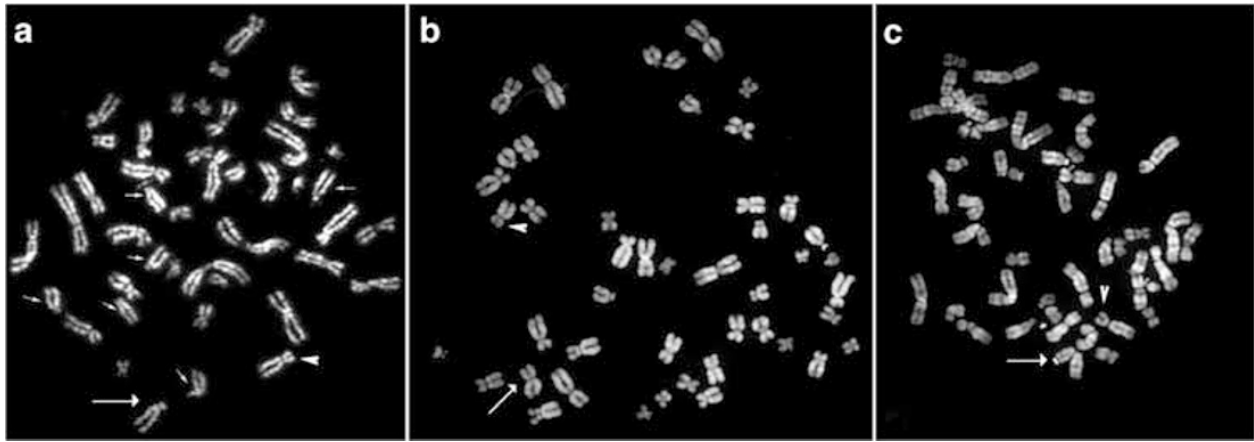

Figure 1 (a) Q-banded metaphase from direct CVS showing the $\operatorname{del}(8)(\mathrm{p} 11)$ (arrow) and the normal chromosome (arrowhead). The six small arrows indicate the D-group chromosomes. (b) Q-banded metaphase from cultured CVS showing the inv dup (8p) (arrow) and the normal chromosome (arrowhead). (c): Q-banded metaphase from cultured CVS showing the inv $\operatorname{dup}(8 p)$ (arrow) with satellites at the tip of its short arm.
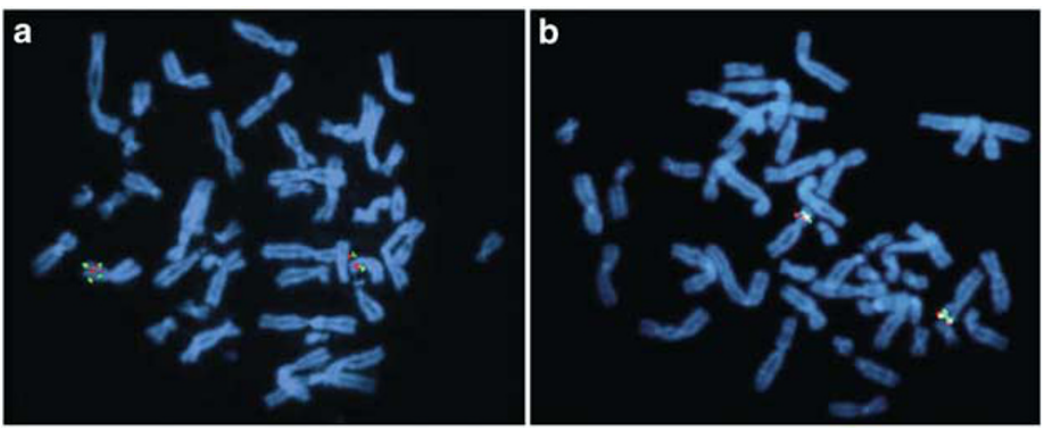

Figure 2 (a) In situ hybridization from a direct CVS metaphase with clones RP11-135I5 (green) and RP11-13N12 (red) demonstrating the inverted duplication. (b) In situ hybridization methaphase from the mother showing the inversion polymorphism with clones RP11-589N15 (green) and RP11-399J23 (red).

counted and in all of them the $\operatorname{del}(8)$ was always substituted by the inv dup $(8 \mathrm{p})$; no other metaphases with the inv dup(8p) ending with fluorescent satellites were found. To exclude the presence of subtelomere probes specific to other chromosomes at the tip of the inv dup(8p), FISH was carried out demonstrating that no subtelomeric probes were translocated at the tip of the inv dup $(8 \mathrm{p})$. Polymorphic markers confirmed that the der(8) was an inv dup (8p) proving the presence of an 8 pter deletion and that of duplicated alleles at $8 \mathrm{p} 21-\mathrm{p} 22$. This analysis also showed the maternal origin of the rearrangement (Figure 3). The final interpretation of the cytogenetic analysis on direct CVS and cultured CVS was: 46,XX, del(8)(p11.2)[26]/46,XX,inv dup(8p)[98] with three of the inv dup(8p) metaphases having a satellite translocated to the short arm of the inv dup $(8 \mathrm{p})$. Both parents had a normal karyotype on 550 bands. FISH with probes RP11399J23 (AC068353) and RP11-589N15 (AC025857) at $3.5 \mathrm{Mb}$ between each other, performed on prometaphases and metaphases from the mother, revealed the presence of the cryptic heterozygous inversion between REPP and REPD (Figure 2b).

\section{Discussion}

We describe the case of a 12-week fetus with severe echographic anomalies showing an $8 \mathrm{p}$ deletion in the direct CVS and an inv dup(8p) in the cultured CVS. We could not exclude that the inv dup $(8 p)$ cell line was present in the direct CVS, since only 26 metaphases could be examined. On the contrary, in the cultured CVS, the absence of the del(8p) cell line was demonstrated through the analysis of 98 metaphases. The rearrangement originated at the maternal meiosis as demonstrated by the presence of two maternal and one paternal allele at some loci of the duplicated region of the inv dup(8p) (see Table 1) and the mother had the typical heterozygous inversion predisposing to the inv dup $(8 \mathrm{p})$ rearrangement. ${ }^{2}$

The finding of different cell lines with a trisomy in direct CVS and normal cells (either with uniparental disomy or really normal) in cultured CVS is fairly common, possibly reflecting the origin of the mosaicism from the correction of a trisomic conception. ${ }^{20}$ A similar situation of selecting the most viable cell line might have occurred in our case. In fact, there is no doubt that $\operatorname{del}(8)(\mathrm{p} 11.2)$ fetuses are less viable than the inv $\operatorname{dup}(8 \mathrm{p})$ ones since no living subjects 


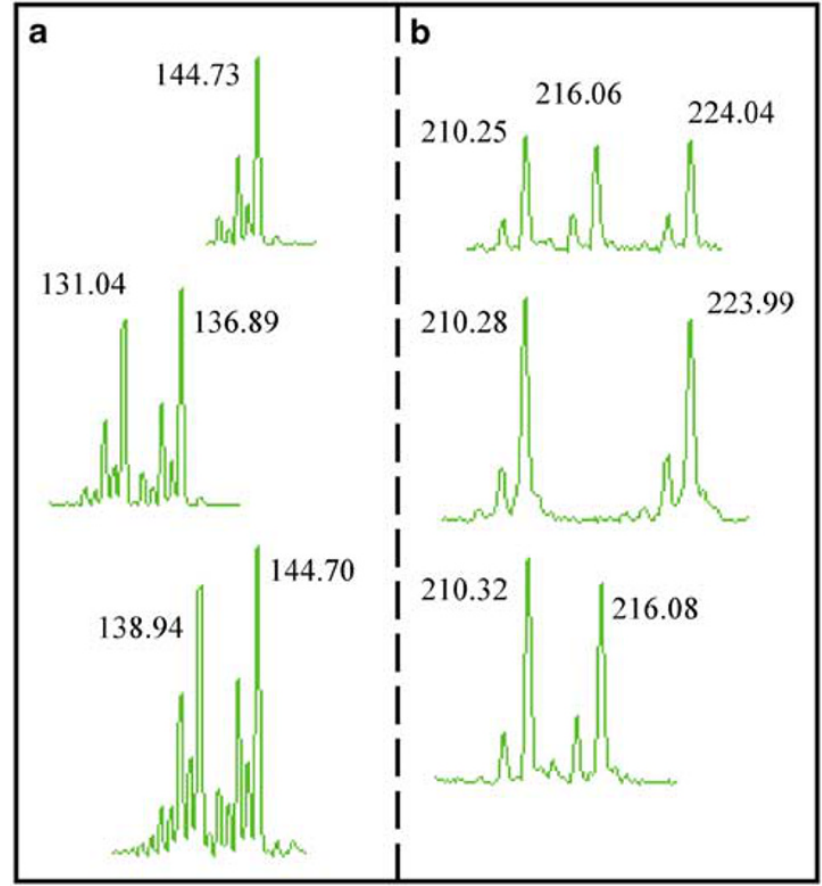

Figure 3 (a) Polymorphic marker D8S1742 showing the deletion on DNA from direct CVS. (b) Polymorphic marker D8S1786 showing the duplication on DNA from cultured CVS. From the top: fetus, mother, father. Both findings indicate that the rearrangement is maternal in origin.

have ever been reported with such an $8 p$ deletion, whereas several cases of inv dup $(8 \mathrm{p}) \mathrm{s}$ are reported, most if not all of them without life-threatening malformations. ${ }^{16}$ Although it is impossible to say whether the mosaicism we found is a confined placental mosaicism (CPM) with only inv dup(8p) cells present in the fetus, its cardiac abnormalities might indeed suggest the influence of the $8 p$ deletion cell line on embryo development. Heart defects are rather rare in inv dup(8p) cases, whereas they are quite common in deletion of the terminal end of chromosome $8 p$ typically in the form of atrial ventricular septal defects. ${ }^{21,22}$ This type of defect in two large unrelated families provided strong evidence for the involvement of GATA4 at $8 \mathrm{p} 23.1 .^{23}$ GATA4 is in single copy in the inv dup(8p) chromosomes, ${ }^{2}$ whereas it should be deleted in the cell line with the del(8p). In fact, the fetus we studied had a cardiac septal defect.

Mosaicisms similar to that described here have been reported three times, twice in two children with dysmorphic features and mental retardation ${ }^{18,19}$ and a third one in prenatal diagnosis. ${ }^{17}$ In the latter case, a mosaic karyotype 46,XX,i(8q) /46,XX, del(8)(p11.2) was found in direct CVS, whereas in the follow-up amniocentesis only metaphases with the inv $\operatorname{dup}(8 \mathrm{p})$ had been found. The authors suggested that the mosaicism $\operatorname{del}(8)(\mathrm{p} 11.2) / \mathrm{inv}$ dup $(8 \mathrm{p})$ derives from the postzygotic breakage of a dicentric chromosome 8 formed through unequal recom- bination of the two pairs of olfactory receptor gene-clusters at $8 \mathrm{p} 23.1$. Vermeesh et al ${ }^{18}$ found the mosaicism $\operatorname{del}(8) / \mathrm{inv}$ $\operatorname{dup}(8 p)$ in the lymphocytes of their patient but not in the fibroblasts where only the inv $\operatorname{dup}(8 \mathrm{p})$ was present. Their molecular analysis demonstrated that the del(8) contained a portion of $8 \mathrm{p}$ present also in the inv $\operatorname{dup}(8 \mathrm{p})$, thus, demonstrating that they were not the one mutual of the other as expected if they were formed by breakage of a dicentric chromosome. Based on this observation, they concluded that the inv $\operatorname{dup}(8 \mathrm{p})$ originated at meiosis, whereas the $8 \mathrm{p}$ - originated by an independent terminal deletion event. We think that the inheritance of a dicentric chromosome to the zygote might indeed explain all the mosaicisms found in the published cases and in our case. We have only to assume that the dicentric chromosome is maintained at least in the cells of the blastula, then undergoing different breakages in different cells leading to inv $\operatorname{dup}(8 \mathrm{p}) \mathrm{s}$ with different duplicated regions and to $\operatorname{del}(8 p) s$ with different deleted regions. Since telomerase activity is present in the early stages of fetal development, ${ }^{24}$ the broken derivatives of the dicentric chromosome can be healed. Cellular selection favouring the cells with a less severe aneuploidy over those with large duplications or deletions is expected to occur as it is known to occur against trisomic cells. ${ }^{25-28}$ The isochromosome 8 in some cells of Soler's case ${ }^{17}$ and the three cells of our inv dup(8p) having satellites at its tip seem to indicate that the dicentric 8 with both the centromeres still active could have been maintained for a certain duration along embryogenesis. In fact, it seems likely to assume that the isochromosome was formed through the fusion of the two chromatids in a neoformed deleted chromosome 8 , which could not be healed by the telomerase any more due to the switch-off of its activity. Similarly, the satellited inv $\operatorname{dup}(8 p)$ might have been formed later in respect to the nonsatellited inv dup(8p) so that it was stabilized by the telomere capture of the short arm of a D- or a Gchromosome. The same situation was reported in an inv $\operatorname{dup}(8 \mathrm{p})$ stabilized by telomere capture from $18 \mathrm{q} .^{29}$

The importance of the findings we discussed here relies on the fact that such mosaic situations with two or more cell lines, all with a different abnormality of the same chromosome, might not be rare. Beyond the three cases with the inv $\operatorname{dup}(8 \mathrm{p}) / \operatorname{del}(8 \mathrm{p})$ mosaicism, ${ }^{17-19}$ we were able to find in the literature four $5 p-/ 5 p+$ cases $\left({ }^{10}\right.$; one studied by us) and several cases with a mosaicism consisting of a cell line with a dicentric isochromosome and another one with a deleted chromosome. Examples are three $18 p-/ 18 p+$ cases, $^{30-32}$ one $18 q-/ 18 q+$ case, $^{33}$ two $\mathrm{Xq}-/ \mathrm{Xq}+$ cases, one of them associated also with a $45, \mathrm{X}$ cell line, ${ }^{34,35}$ several cases of dicentric Ypter-q11 that are frequently mosaic for a del(Y)(pter-q11) cell line. ${ }^{16}$

In conclusion, our findings suggest that (1) mosaicism might be rather common in chromosome rearrangements consisting of inversion duplication associated with distal 
deletion, that is, in those rearrangements having a dicentric chromosome as the first product of the abnormal meiotic recombination; (2) the timing of the dicentric breakage may be placed in early embryogenesis rather than at the meiosis II and (3) phenotype/karyotype relationship in inv dup rearrangements must also take into account the influence of the other abnormal cell line during embryogenesis.

\section{Acknowledgements}

This work was supported by cofin02-and cofinO3-MIUR (to OZ), the Italian Telethon Foundation (GP0247Y01 to OZ) and the Cariplo Foundation.

\section{References}

1 Floridia G, Piantanida M, Minelli A et al: The same molecular mechanism at the maternal meiosis I produces mono- and dicentric 8p duplications. Am J Hum Genet 1996; 58: 785-796.

2 Giglio S, Broman KW, Matsumoto N et al: Olfactory receptor-gene clusters, genomic-inversion polymorphisms, and common chromosome rearrangements. Am J Hum Genet 2001; 68: $874-883$.

3 Mewar R, Harrison W, Weaver DD, Palmer C, Davee MA, Overhauser J: Molecular cytogenetic determination of a deletion/duplication of $1 \mathrm{q}$ that results in a trisomy 18 syndrome-like phenotype. Am J Med Genet 1994; 52: 178-183.

4 De Brasi D, Rossi E, Giglio S et al: Inv dup del (1)(pter$>\mathrm{q} 44:: \mathrm{q} 44->\mathrm{q} 42:$ ) with the classical phenotype of trisomy 1q42-qter. Am J Med Genet 2001; 104: 127-130.

5 Bonaglia MC, Giorda R, Poggi G et al: Inverted duplications are recurrent rearrangements always associated with a distal deletion: description of a new case involving 2q. Eur J Hum Genet 2000; 8: $597-603$.

6 Jenderny J, Poetsch M, Hoeltzenbein M, Friedrich U, Jauch A: Detection of a concomitant distal deletion in an inverted duplication of chromosome 3 . Is there an overall mechanism for the origin of such duplications/deficiencies? Eur J Hum Genet 1998; 6: 439-444.

7 Kennedy D, Silver MM, Winsor EJ et al: Inverted duplication of the distal short arm of chromosome 3 associated with lobar holoprosencephaly and lumbosacral meningomyelocele. Am J Med Genet 2000; 91: 167-170.

8 Cotter PD, Kaffe S, Li L, Gershin IF, Hirschhorn K: Loss of subtelomeric sequence associated with a terminal inversion duplication of the short arm of chromosome 4. Am J Med Genet 2001; 102: 76-80.

9 Sreekantaiah C, Kronn D, Marinescu RC, Goldin B, Overhauser J: Characterization of a complex chromosomal rearrangement in a patient with a typical catlike cry and no other clinical findings of cri-du-chat syndrome. Am J Med Genet 1999; 86: 264-268.

10 Perfumo C, Cerruti Mainardi P, Cali A et al: The first three mosaic cri du chat syndrome patients with two rearranged cell lines. J Med Genet 2000; 37: 967-972.

11 Teebi AS, Gibson L, McGrath J, Meyn MS, Breg WR, Yang-Feng TL: Molecular and cytogenetic characterization of $9 \mathrm{p}$ - abnormalities. Am J Med Genet 1993; 46: 288-292.

12 Hoo JJ, Chao M, Szego K, Rauer M, Echiverri SC, Harris C: Four new cases of inverted terminal duplication: a modified hypothesis of mechanism of origin. Am J Med Genet 1995; 58: 299-304.

13 Moog U, Engelen JJ, de Die-Smulders CE et al: Partial trisomy of the short arm of chromosome 18 due to inversion duplication and direct duplication. Clin Genet 1994; 46: 423-429.

14 Courtens W, Grossman D, Van Roy N et al: Noonan-like phenotype in monozygotic twins with a duplication-deficiency of the long arm of chromosome 18 resulting from a maternal paracentric inversion. Hum Genet 1998; 103: 497-505.

15 Pangalos C, Theophile D, Sinet PM et al: No significant effect of monosomy for distal 21q22.3 on the Down syndrome phenotype in 'mirror' duplications of chromosome 21. Am J Hum Genet 1992; 51: $1240-1250$

16 Schinzel A: Catalogue of unbalanced chromosome aberrations in man, 2nd edn. Berlin; New York: de Gruyter, 2001.

17 Soler A, Sanchez A, Carrio A, Badenas C, Mila M, Borrell A: Fetoplacental discrepancy involving structural abnormalities of chromosome 8 detected by prenatal diagnosis. Prenat Diagn 2003; 23: $319-322$.

18 Vermeesch JR, Thoelen R, Salden I, Raes M, Matthijs G, Fryns JP: Mosaicism del (8p)/inv dup(8p) in a dysmorphic female infant: a mosaic formed by a meiotic error at the $8 \mathrm{p}$ OR gene and an independent terminal deletion event. J Med Genet 2003; 40: e93.

19 van Balkom ID, Hagendoorn J, De Pater JM, Hennekam RC: Partial monosomy $8 \mathrm{p}$ and partial trisomy $8 \mathrm{p}$ with moderate mental retardation. Genet Couns 1992; 3: 83-89.

20 Wolstenholme J: Confined placental mosaicism for trisomies 2, 3, $7,8,9,16$, and 22: their incidence, likely origins, and mechanisms for cell lineage compartmentalization. Prenat Diagn 1996; 16: 511-524.

21 Devriendt K, Matthijs G, Van Dael R et al: Delineation of the critical deletion region for congenital heart defects, on chromosome 8p23.1. Am J Hum Genet 1999; 64: 1119-1126.

22 Giglio S, Graw SL, Gimelli G et al: Deletion of a 5-cM region at chromosome $8 \mathrm{p} 23$ is associated with a spectrum of congenital heart defects. Circulation 2000; 102: 432-437.

23 Garg V, Kathiriya IS, Barnes R et al: GATA4 mutations cause human congenital heart defects and reveal an interaction with TBX5. Nature 2003; 424: 443-447.

24 Wright WE, Piatyszek MA, Rainey WE, Byrd W, Shay JW: Telomerase activity in human germline and embryonic tissues and cells. Dev Genet 1996; 18: 173-179.

25 Wolstenholme J, Rooney DE, Davison EV: Confined placental mosaicism, IUGR, and adverse pregnancy outcome: a controlled retrospective U.K. collaborative survey. Prenat Diagn 1994; 14 $345-361$.

26 Kalousek DK, Vekemans M: Confined placental mosaicism. J Med Genet 1996; 33: 529-533.

27 Robinson WP, Barrett IJ, Bernard L et al: Meiotic origin of trisomy in confined placental mosaicism is correlated with presence of fetal uniparental disomy, high levels of trisomy in trophoblast, and increased risk of fetal intrauterine growth restriction. Am J Hum Genet 1997; 60: 917-927.

28 Robinson WP: Mechanisms leading to uniparental disomy and their clinical consequences. BioEssays 2000; 22: 452-459.

29 Kostiner DR, Nguyen H, Cox VA, Cotter PD: Stabilization of a terminal inversion duplication of $8 p$ by telomere capture from 18q. Cytogenet Genome Res 2002; 98: 9-12.

30 Badalian LO, Mutovin GR, Malygina NA, Petrukhin AS: Rare case of mosaicism for chromosome 18, karyotype: 46, XX, del(18) (p11)/46, XX, i(18q). Genetika 1983; 19: 1912-1915.

31 Sutton SD, Ridler MA: Prenatal detection of monosomy 18p and trisomy $18 \mathrm{q}$ mosaicism with unexpected fetal phenotype. J Med Genet 1986; 23: 258-259.

32 Stephen GS, Couzin DA, Watt JL, Rankin R: Prenatal diagnosis of a case of 46,XY,18p-/46,XY,18p+ mosaicism. Prenat Diagn 1989; 9: $57-60$.

33 Ausems MG, Bhola SL, Post-Blok CA, Hennekam RC, de France HF: $18 \mathrm{q}-$ and $18 \mathrm{q}+$ mosaicism in a mentally retarded boy. Am J Med Genet 1994; 53: 296-299.

34 Morichon-Delvallez N, Couturier J, Bourdrel V: Inherited Xq duplication due to a zygotic translocation $\mathrm{t}(\mathrm{X} ; \mathrm{X})(\mathrm{q} 23 ; \mathrm{q} 27)$. Ann Genet 1988; 31: 117-119.

35 Calvano S, de Cillis GP, Croce AI, Perla G, Notarangelo A, Zelante L: A complex mosaicism 45,X/46,X,del(Xq)/46,X,idic(Xq) in a patient with secondary amenorrhea. Ann Genet 2002; 45: 137-140. 